\title{
APPROXIMATION IN FUNCTION MODULES
}

\section{Fowzi Ahmed Sejeeni}

We investigate the existence of best approximation of an element $\alpha$ in a function module from a subfunction module whose fibers satisfy the intersection property of balls. Also we investigate the lower semicontinuity of the metric projection associated with such a subfunction module.

\section{INTRODUCTION}

Let $E$ be a normed linear space and $G$ a closed subspace of $E$. The set

$$
\mathrm{P}_{G}(x)=\left\{g_{0} \in G:\left\|x-g_{0}\right\|=\inf \|x-g\|, g \in G\right\}
$$

is called the set of all best approximations to $x$ from $G$. This defines a set valued mapping $P_{G}$ which is called the metric projection onto $G$. A mapping $s: E \rightarrow G$ is called a selection for $P_{G}$ if $s(x) \in P_{G}(x)$ for all $x \in E$. A subspace $G$ of a normed linear space $E$ is called proximinal (respectively Chebychev) if $P_{G}(x)$ contains at least (exactly) one element for all $x \in E$.

The set valued mapping $P_{G}$ is called lower semicontinuous (l.s.c.) if the set

$$
\left\{x \in E: P_{G}(x) \cap U \neq \emptyset\right\}
$$

is open for each open subset $U$ of $G$ or, what is equivalent, for each sequence $\left\{x_{n}\right\}$ in $E$ converging to $x$ in $E$ and for each $g$ in $P_{G}(x)$, there is a sequence $\left\{g_{n}\right\}$ in $G$ such that for each $n \in N, g_{n} \in P_{G}\left(x_{n}\right)$ and $g_{n} \rightarrow g$, see $[2$, p.365].

Definition 1.1: A subspace $G$ of the Banach space $E$ is said to have the twoball property for open balls if for any pair $B\left(x_{1}, r_{1}\right)$ and $B\left(x_{2}, r_{2}\right)$ of open balls such that $B\left(x_{1}, r_{1}\right) \cap B\left(x_{2}, r_{2}\right) \neq \emptyset$ and $B\left(x_{i}, r_{i}\right) \cap G \neq \emptyset$ for $i=1,2$, the intersection $\left(G \cap B\left(x_{1}, r_{1}\right) \cap B\left(x_{2}, r_{2}\right)\right)$ is nonvoid; see [1, Definition 2.16].

Let $T$ be a nonvoid compact Hausdorff space and $\left(E_{t}\right)$ a family of Banach spaces over $T$. Consider the Banach space $\prod_{t \in T}^{\infty} E_{t}=\left\{\alpha \in \prod_{t \in T} E_{t}:\|\alpha\|_{\infty}=\sup _{t \in T}\|\alpha(t)\|_{t}<\infty\right\}$ (where $\|\cdot\|_{t}$ is the norm on the Banach space $E_{t}$ ). Closed subspaces of $\prod_{t \in T}^{\infty} E_{t}$ will be called Banach spaces of a vector valued function on $T$.

Received 30 April 1990

Copyright Clearance Centre, Inc. Serial-fee code: 0004-9729/91 \$A2.00+0.00. 
Definition 1.2: A function module is a triple $\left(T,\left(E_{t}\right)_{t \in T}, E_{\infty}\right)$, where $T$ is a nonvoid compact Hausdorff space (called base space), $\left(E_{t}\right)_{t \in T}$ a family of Banach spaces (the component spaces) and $E_{\infty}$ a closed subspace of the space $\prod_{t \in T}^{\infty} E_{t}$ such that:

(1) $E_{\infty}$ is a $C(T)$-module (where $C(T)$ is the Banach algebra of all continuous scalar valued functions on $T),(f, \alpha)(t)=f(t) \alpha(t), f \in C(T)$, $\alpha \in E_{\infty}$.

(2) For every $\alpha \in E_{\infty}$, the map $t \mapsto\|\alpha(t)\|_{t}$ is upper semicontinuous.

(3) $E_{t}=\left\{\alpha(t): \alpha \in E_{\infty}\right\}$ for every $t \in T$.

(4) $\left\{t: t \in T, E_{t} \neq\{0\}\right\}=T$.

REMARK. Instead of " $\left(T,\left(E_{t}\right)_{t \in T}, E_{\infty}\right)$ is a function module" we will often say that $E_{\infty}$ is a function module in $\prod_{t \in T}^{\infty} E_{t}$ or (if $T$ and $\left(E_{t}\right)_{t \in T}$ are understood) that $E_{\infty}$ itself is a function module, see [1, Definition 4.1].

Definition 1.3: Let $G_{\infty}$ be a sub-function module in the function module $E_{\infty}$ and $\alpha$ be an element of $E_{\infty}$. The element $\gamma_{0}$ in $G_{\infty}$ is called:

(1) global best approximation of $\alpha$ from $G_{\infty}$ if

$$
\left\|\alpha-\gamma_{0}\right\|_{\infty}=\inf \left\{\|\alpha-\gamma\|_{\infty}: \gamma \in G_{\infty}\right\} ;
$$

(2) local best approximation if for each $t \in T$

$$
\left\|\alpha(t)-\gamma_{0}(t)\right\|_{t}=\inf \left\{\|\alpha(t)-g\|_{t}: g \in G_{t}\right\}
$$

that is, $\gamma(t)$ in $P_{G_{t}}(\alpha(t))$ for each $t \in T$.

Note that local best approximations are always global but the converse is not always true.

\section{RESUlts}

THEOREM 2.1. Let $G$ be a closed subspace of the Banach space $E$. If $G$ satisfies the two-ball property for open balls, then $G$ is proximinal and $\mathrm{P}_{G}$ is lower semicontinuous.

Proof: Let $x$ be an arbitrary element in $E \backslash G$ and define $r=d(x, G)=$ $\inf \{\|x-g\|: g \in G\}$. For any positive real number $\varepsilon$ and any $g \in G$ such that $\|x-g\|<r+\varepsilon$ we have $B(x, r+\varepsilon / 2) \cap B(g, \varepsilon / 2) \neq \emptyset, B(x, r+\varepsilon / 2) \cap G \neq \emptyset$ and $B(g, \varepsilon / 2) \cap G \neq \emptyset$. Therefore $B(x, r+\varepsilon / 2) \cap B(g, \varepsilon / 2) \cap G \neq \emptyset$, and then there exists an element $g_{0}$ in $G$ satisfying the following:

$$
\left\|x-g_{0}\right\| \leqslant r+\frac{\varepsilon}{2} \text { and }\left\|g-g_{0}\right\| \leqslant \frac{\varepsilon}{2} .
$$


By applying (2.1) inductively, we can construct a sequence $\left\{g_{n}\right\}$ in $G$ satisfying the following:

$$
\left\|x-g_{n}\right\| \leqslant r+2^{-n} \text { and }\left\|g_{n}-g_{n+1}\right\| \leqslant 2^{-n}
$$

The sequence $\left\{g_{n}\right\}$ is Cauchy, and hence it has a limit $g$ in $G$. Moreover, we have $\|x-g\|=r$; that is, $g \in P_{G}(x)$.

For the lower semi-continuity of $\mathrm{P}_{G}$, let $U$ be an arbitrary open subset of $G$ and $V=\left\{x \in E: P_{G}(x) \cap U \neq \emptyset\right\}$. We may assume without loss of generality that $V \neq \emptyset$ and show that $V^{c}$ (the complement of $V$ in $E$ ) is closed. For, let $\left\{x_{n}\right\}$ be a sequence in $V^{c}$ converging to $x$ in $V, g \in P_{G}(x) \cap U$ and $\varepsilon>0$ such that $B(g, \varepsilon) \subseteq U$. Define $r_{n}=d\left(x_{n}, G\right)$ and $r=d(x, G)$. Let $N$ be the positive integer such that $\left\|x_{n}-x\right\|<\varepsilon / 2$ and $\left\|r_{n}-r\right\|<\varepsilon / 2$ for each $n \geqslant N$. Now, for each $n \geqslant N$, the two balls $B\left(x_{n}, r_{n}\right)$ and $B(g, \varepsilon)$ satisfy the following:

$$
B\left(x_{n}, r_{n}\right) \cap B(g, \varepsilon) \neq \emptyset
$$

(since $\left\|x_{n}-g\right\| \leqslant\left\|x_{n}-x\right\|+\|x-g\|<\varepsilon / 2+r<\varepsilon / 2+\left(r_{n}+\varepsilon / 2\right)=r_{n}+\varepsilon$,) $B\left(x_{n}, r_{n}\right) \cap G \neq \emptyset$, and $B(g, \varepsilon) \cap G \neq \emptyset$. Hence $B\left(x_{n}, r_{n}\right) \cap B(g, \varepsilon) \cap G \neq \emptyset$, or, what is equivalent, there is a $g_{n} \in \mathrm{P}_{G}\left(x_{n}\right)$ such that $g_{n} \in B(g, \varepsilon) \subseteq U$. This contradicts the assumption. Thus $V$ must be open.

THEOREM 2.2. Let $E_{\infty}$ be a function module in $\prod_{t \in T}^{\infty} E_{t}$, such that for each $\alpha$ in $E_{\infty}$ the mapping $t \mapsto\|\alpha(t)\|_{t}$ is continuous. If $\alpha_{1}, \ldots, \alpha_{n}$ are elements of $E_{\infty}$ such that for each $t$ in $T, \operatorname{span}\left\langle\alpha_{1}(t), \ldots, \alpha_{n}(t)\right\rangle$ has dimension $n$ and satisfies the two-ball property for open balls, then $\operatorname{span}\left\langle\alpha_{1}, \ldots, \alpha_{n}\right\rangle$ contains a local best approximation for each $\alpha \in E_{\infty}$.

In order to prove the above theorem, we need the following lemma, which perhaps is interesting in itself.

Lemma 2.3. With the assumption of Theorem 2.2, for each $\alpha$ in $E_{\infty}$ the function $\rho: T \rightarrow \mathbf{R}$ defined by $\rho(t)=d\left(\alpha(t), G_{t}\right)$ is continuous.

Proof: Let $H: T \times \ell_{1}^{n} \rightarrow \mathrm{R}$ be the mapping defined by $H(t, a)=$ $\left\|\alpha(t)-\sum_{i=1}^{n} a_{i} \alpha_{i}(t)\right\|_{t}$ (where $a_{i}=h_{i}(a) a n d\left\{h_{i}\right\}$ is the sequence of coefficient functionals associated with the unit vector basis of $\left.\ell_{1}^{n}\right)$. Let $\left(t_{0}, r\right)$ be a fixed point in $T \times \ell_{1}^{n}$. 
Then

$$
\begin{aligned}
\left|H(t, a)-H\left(t_{0}, r\right)\right| \leqslant|H(t, a)-H(t, r)|+\left|H(t, r)-H\left(t_{0}, r\right)\right| & \\
\leqslant \sum_{i=1}^{n}\left|a_{i}-r_{i}\right|\left\|\alpha_{i}(t)\right\|_{t} & \quad+\left|\left\|\alpha(t)-\sum_{i=1}^{n} r_{i} \cdot \alpha_{i}(t)\right\|_{t}-\left\|\alpha\left(t_{0}\right)-\sum_{i=1}^{n} r_{i} \cdot \alpha_{i}\left(t_{0}\right)\right\|_{t_{0}}\right| .
\end{aligned}
$$

This inequality and the continuity of the map $t \mapsto\left\|\alpha(t)-\sum_{i=1}^{n} r_{i} \cdot \alpha_{i}(t)\right\|_{t}$ imply that $H$ is continuous on $T$. For each $t \in T$, define $\Lambda_{t}: \ell_{1}^{n} \rightarrow G_{t}$ by $a \mapsto \sum_{i=1}^{n} a_{i} \cdot \alpha_{i}(t)$. Here $\Lambda_{t}$ is a one to one onto linear mapping. Moreover for each $t \in T, a \in \ell_{1}^{n}$ we have

$$
\begin{aligned}
\left\|\Lambda_{t} a\right\|_{t} & =\left\|\sum_{i=1}^{n} a_{i} \cdot \alpha_{i}(t)\right\|_{t} \leqslant \sum_{i=1}^{n}\left\|a_{i}\right\|\left\|\alpha_{i}(t)\right\|_{t} \\
& \leqslant n \max _{i}\left\|h_{i}\right\| \cdot \max _{i}\left\|\alpha_{i}\right\|_{\infty} .
\end{aligned}
$$

Hence the open mapping theorem and the uniform boundedness principle give positive reals $m$ and $k$ such that

$$
m\|a\| \leqslant\left\|\Lambda_{t} a\right\|_{t} \leqslant k\|a\| \quad \forall t \in T, \quad \forall a \in \ell_{1}^{n} .
$$

Now, let $t_{0}$ be a fixed point in $T$, and $\left\{t_{b}\right\}_{b \in B}$ be any net in $T$ converging to $t_{0}$. Pick $g \in P_{G_{i_{0}}}\left(\alpha\left(t_{0}\right)\right)$ and write $g=\sum_{i=1}^{n} g_{i} . \alpha_{i}\left(t_{0}\right), \beta=\sum_{i=1}^{n} g_{i} \cdot \alpha_{i}\left(\beta \in G_{\infty}=\right.$ span of $\left\langle\alpha_{1}, \ldots, \alpha_{n}\right\rangle$ over $\left.C(T)\right)$. Let $\varepsilon$ be any positive real number, and $U_{t_{0}}$ the neighbourhood of $t_{0}$ such that $\|\alpha(t)-\beta(t)\|_{t}<\left\|\alpha\left(t_{0}\right)-\beta\left(t_{0}\right)\right\|_{t_{0}}+\varepsilon=\rho\left(t_{0}\right)+\varepsilon$. But

$$
\rho(t) \leqslant\|\alpha(t)-\beta(t)\|_{t}<\rho\left(t_{0}\right)+\varepsilon \quad \forall t \in U_{t_{0}}
$$

(since $\left.\beta(t)=\sum_{i=1}^{n} g_{i} \cdot \alpha_{i}(t) \in G_{t}\right)$. The net $\left\{a\left(t_{b}\right)\right\}_{b \in B}$ (where $a\left(t_{b}\right)=\left(a_{1}\left(t_{b}\right), \ldots, a_{n}\left(t_{b}\right)\right.$ ) and $\left.\left\|\alpha\left(t_{b}\right)-\sum_{i=1}^{n} a_{i}\left(t_{b}\right) \cdot \alpha_{i}\left(t_{b}\right)\right\|_{t_{b}}=\rho\left(t_{b}\right)\right)$ is eventually bounded since

$$
\begin{aligned}
\left\|a\left(t_{b}\right)\right\| & \leqslant \frac{1}{m}\left\|\sum_{i=1}^{n} a_{i}\left(t_{b}\right) \alpha_{i}\left(t_{b}\right)\right\|_{t_{b}} \\
& \leqslant \frac{1}{m}\left(\left\|\alpha\left(t_{b}\right)-\sum_{i=1}^{n} a_{i}\left(t_{b}\right) \cdot \alpha_{i}\left(t_{b}\right)\right\|_{t_{b}}+\left\|\alpha\left(t_{b}\right)\right\|_{t_{b}}\right) .
\end{aligned}
$$


By (2.4) there is a $c \in B$ such that $\rho\left(t_{b}\right)<\rho\left(t_{0}\right)+1$ for each $b \geqslant c$. Thus $\left\|a\left(t_{b}\right)\right\| \leqslant$ $(1 / m)\left(\rho\left(t_{0}\right)+1+\|\alpha\|_{\infty}\right)$. We may assume without loss of generality that $a\left(t_{b}\right) \rightarrow a$.

$$
\begin{gathered}
\rho(t)-\rho\left(t_{0}\right) \leqslant H\left(t, a\left(t_{0}\right)\right)+H\left(t_{0}, a\left(t_{0}\right)\right) . \\
\rho\left(t_{0}\right)-\rho(t) \leqslant H\left(t_{0}, a(t)\right)+H(t, a(t)) . \\
\left|H\left(t_{0}, a\left(t_{b}\right)\right)-H\left(t_{b}, a\left(t_{b}\right)\right)\right| \leqslant \\
\quad\left|H\left(t_{0}, a\right)-H\left(t_{0}, a\left(t_{b}\right)\right)\right| \\
+\left|H\left(t_{0}, a\right)-H\left(t_{b}, a\left(t_{b}\right)\right)\right| .
\end{gathered}
$$

The continuity of the map $H$ and $a\left(t_{b}\right) \rightarrow a$ imply that $\left|B\left(t_{0}, a\left(t_{b}\right)\right)-H\left(t_{b}, a\left(t_{b}\right)\right)\right| \rightarrow$ 0 as $t_{b} \rightarrow t$. Consequently, (2.5) and (2.6) imply that $\rho$ is continuous.

Proof of THE Theorem: It suffices to show that for each $\alpha \in E_{\infty}, t_{0} \in T$, $g \in P_{G_{t_{0}}}\left(\alpha\left(t_{0}\right)\right)$ and $\varepsilon>0$ there exists $\gamma \in G_{\infty}$ such that $\gamma\left(t_{0}\right)=g$, and $d\left(\gamma(t), P_{G_{t}}(\alpha(t))\right)<\varepsilon$ for each $t \in T$. Write $g=\sum_{i=1}^{n} g_{i} . \alpha_{i}\left(t_{0}\right), \alpha_{0}=\sum_{i=1}^{n} g_{i} . \alpha_{i} \in G_{\infty}$. Let $U_{t_{0}}$ be the neighbourhood of $t_{0}$ such that, for $s \in U_{t_{0}}$,

and

$$
\begin{aligned}
\| \alpha_{0}(s)-\left.\alpha(s)\right|_{0} & <\left\|\alpha_{0}\left(t_{0}\right)-\alpha\left(t_{0}\right)\right\|_{t_{0}}+\varepsilon / 4 \\
\rho\left(t_{0}\right) & <\rho(s)+\varepsilon / 4 .\left\|\alpha_{0}(s)-\alpha(s)\right\|_{0} \\
& <\left\|\alpha_{0}\left(t_{0}\right)-\alpha\left(t_{0}\right)\right\|_{t_{0}}+\varepsilon / 4 \\
& =\rho\left(t_{0}\right)+\varepsilon / 4<\rho(s)+\varepsilon / 2 .
\end{aligned}
$$

Thus for each $s \in U_{t_{0}}$, we have the following:

and

$$
\begin{aligned}
& B(\alpha(s), \rho(s)) \cap B\left(\alpha_{0}(s), \varepsilon / 2\right) \neq \emptyset \\
& B(\alpha(s), \rho(s)) \cap G, \neq \emptyset \\
& B\left(\alpha_{0}(s), \varepsilon / 2\right) \cap G_{\triangleleft} \neq \emptyset .
\end{aligned}
$$

Therefore, there is $g_{8}$ in $B(\alpha(s), \rho(s)) \cap B\left(\alpha_{0}(s), \varepsilon / 2\right) \cap G_{,}$, and then $d\left(\alpha_{0}(s), \mathrm{P}_{G_{s}}(\alpha(s))\right)$ : $\left\|g_{,}-\alpha_{0}(s)\right\|_{\varepsilon} \leqslant \varepsilon / 2<\varepsilon$.

Now, for each $t \in T, t \neq t_{0}$, select a $g_{t}$ from $P_{G_{t}}(\alpha(t))$. By the above there is $\alpha_{t} \in G_{\infty}$ and a neighbourhood $U_{t}$ of $t$ (we may assume that $U_{t_{0}} \cap U_{t}=\emptyset$, since $T$ is Hausdorff) such that $\alpha_{t}(t)=g_{t}$ and $d\left(\alpha_{t}(p), P_{G_{p}}(\alpha(p))\right)<\varepsilon$ for each $p$ in $U_{t}$. Let $f_{t}: T \rightarrow[0,1]$ be the continuous function such that $f_{\mid u_{t_{0}}}=1$ and $f_{\mid u_{t}}=0$. For $\beta_{t}=f_{t} \cdot \alpha_{0}+\left(1-f_{t}\right) . \alpha_{t}$, we have $\beta_{t \mid u_{t}}=\alpha_{0}, \beta_{t \mid u_{t}}=\alpha_{t}$ and $d\left(\beta_{t}(s), P_{G}(\beta(s))\right)<\varepsilon$ for each $s$ in $V_{t}=U_{t_{0}} \cup U_{t}$. The collection $\left\{V_{t}: t \in T\right\}$ forms an open covering of $T$; then there are $t_{1}, \ldots, t_{n}$ in $T$ such that $T=\bigcup_{i=1}^{n} V_{t_{i}}$. Let $\left\{h_{i}\right\}_{i=1}^{n}$ be the partition of 
unity subordinate to $\left\{V_{t_{i}}\right\}$. A simple calculation will show that $\gamma=\sum_{i=1}^{n} h_{i} \cdot \beta_{t_{i}}$ is the desired element of $G_{\infty}$. Since $\varepsilon$ was arbitrary, the result follows from the closeness of $P_{G_{t}}(\alpha(t))$ and the fact that $d\left(\alpha(t), G_{t}\right) \leqslant d\left(\alpha, G_{\infty}\right)$ for each $t$ in $T$.

TheOREM 2.4. Let $E_{\infty}$ be a function module in $\prod_{t \in T}^{\infty} E_{t}$. If $G_{\infty}$ is a sub-C(T)module of $E_{\infty}$ such that for each $t$ in $T$ the fiber $G_{t}=\left\{\gamma(t): \gamma \in G_{\infty}\right\}$ has the two-ball property for open balls, then $G_{\infty}$ is proximinal (global best approximation exists).

Proof: Let $\alpha$ be any fixed element of $E_{\infty}$. For each $t$ in $T$, let $g(t) \in P_{G_{t}}(\alpha(t))$. Define

$$
r=\inf _{\gamma \in G_{\infty}}\|\alpha-\gamma\|_{\infty} \geqslant \sup _{t \in T}\|\alpha(t)-g(t)\|_{t} .
$$

We shall show that there is a Cauchy sequence $\left\{\gamma_{n}\right\}$ in $G_{\infty}$ such that $\left\|\alpha-\gamma_{n}\right\|_{\infty} \rightarrow r$. For, let $\varepsilon>0$; then by definition of $r$ there is $\beta \in G_{\infty}$ such that $\|\beta-\alpha\|_{\infty}<r+\varepsilon$. We will show that there is another element $\gamma \in G_{\infty}$. such that

$$
\|\alpha-\gamma\|_{\infty}<r+\frac{\varepsilon}{2} \text { and }\|\beta-\gamma\|_{\infty} \leqslant \frac{\varepsilon}{2} .
$$

For each $t \in T$, the two balls $B(\alpha(t), r)$ and $B(\beta(t), \varepsilon)$ satisfy the conditions of the two-ball property (since $\|\alpha(t)-g(t)\|_{t} \leqslant r$ and $\|\beta(t)-\alpha(t)\|_{t} \leqslant\|\beta-\alpha\|_{\infty}<$ $r+\varepsilon)$. Let $x(t) \in G_{t}$ be such that $\|\alpha(t)-x(t)\|_{t} \leqslant r$ and $\|\beta(t)-x(t)\|_{t}<\varepsilon$. Put $y_{t}=(x(t)+\beta(t)) / 2 ;$ then

$$
\begin{gathered}
\|\alpha(t)-y(t)\|_{t} \leqslant\|\alpha(t)-x(t)\|_{t}+\|x(t)-y(t)\|_{t}<r+\varepsilon / 2 \text { and } \\
\|\beta(t)-y(t)\|_{t}<\varepsilon / 2 .
\end{gathered}
$$

Now, let $\gamma_{t} \in G_{\infty}$ be such that $\gamma_{t}(t)=y_{t}$ and $V_{t}$ the neighbourhood of $t$ such that for each $s$ in $U_{t}$

$$
\left\|\alpha(s)-\gamma_{t}(s)\right\|_{0}<r+\varepsilon / 2 \text { and }\left\|\beta(s)-\gamma_{t}(s)\right\|_{\varepsilon}<\frac{\varepsilon}{2} ;
$$

(such $U_{t}$ exists by (u.s.c.) of the norm functions). The collection $\left\{U_{t}: t \in T\right\}$ forms an open covering of $T$. Let $t_{1}, \ldots, t_{n}$ be in $T$ such that $T=\bigcup_{i=1}^{n} U_{t_{i}}$ and $\left\{f_{i}\right\}_{i=1}^{n}$ the partition of unity subordinate to $\left\{U_{t_{i}}\right\}_{i=1}^{n}$. A simple calculation will show that $\gamma=\sum_{i=1}^{n} f_{i} \cdot \gamma_{t_{i}}$ is the desired element.

By applying (2.10) inductively, we can construct a sequence $\left\{\gamma_{n}\right\}$ in $G_{\infty}$ such that

$$
\left\|\alpha-\gamma_{n}\right\|_{\infty} \leqslant r+2^{-n} \text { and }\left\|\gamma_{n}-\gamma_{n+1}\right\|_{\infty} \leqslant 2^{-n}
$$


The second inequality of (2.11) implies that $\left\{\gamma_{n}\right\}$ is Cauchy; hence it has a limit $\gamma_{0}$ in $G_{\infty}$ and the first inequality of (2.11) implies that $\|\alpha-\gamma\|_{\infty}=r$; that is, $\gamma$ in $P_{G_{\infty}}(\alpha)$.

THEOREM 2.5. Let $E_{\infty}$ be a function module in $\prod_{t \in T}^{\infty} E_{t}$. If $G_{\infty}$ is a sub-C(T)module of $E_{\infty}$ such that for each $t$ in $T$ the fiber $G_{t}=\left\{\gamma(t): \gamma \in G_{\infty}\right\}$ has the two-ball property for open balls, then $\mathbf{P}_{G_{\infty}}$ is (l.s.c.).

In order to prove the above theorem, we need the following lemma, which maybe is interesting in itself.

Lemma 2.6. With the assumption of Theorem 2.5, for each $\alpha$ in $E_{\infty}, t$ in $T$ and $x_{t}$ in $G_{t}$ such that $\left\|\alpha(t)-x_{t}\right\|_{t} \leqslant r=d\left(\alpha, G_{\infty}\right)$, there is $\gamma$ in $P_{G_{\infty}}(\alpha)$ such that $\gamma(t)=x_{t}$.

Proof: We shall show that for each positive $\varepsilon$ there are two elements $\beta_{\varepsilon}$ and $\gamma_{\varepsilon}$ in $G_{\infty}$ such that

$$
\begin{gathered}
\beta_{\varepsilon}(t)=\gamma_{e}(t)=x_{i} ; \\
\left\|\alpha-\beta_{\varepsilon}\right\|_{\infty}<r+\varepsilon ; \\
\left\|\alpha-\gamma_{e}\right\|_{\infty}<r+\frac{\varepsilon}{2} \text { and }\left\|\beta_{\varepsilon}-\gamma_{\varepsilon}\right\|_{\infty}<\frac{\varepsilon}{2} .
\end{gathered}
$$

To see this, let $s \in T, x_{a} \in G_{\text {s }}$ such that $\left\|x_{a}-\alpha(s)\right\|_{a} \leqslant r$ (if $s=t$ take $x_{x}=x_{t}$ ). Let $\varphi, \varphi$, be the elements of $G_{\infty}$ such that $\varphi(t)=x_{t}$ and $\varphi_{s}(s)=x_{s}$ and $h: T \longrightarrow[0,1]$ the continuous function such that $h(t)=0$ and $h(s)=1$. Take $\beta_{s}=(1-h) \cdot \varphi+h . \varphi$ s and let $U$, be the neighbourhood of $s$ such that $\left\|\alpha(p)-\beta_{s}(p)\right\|_{p}<r+\varepsilon$ for each $p$ in $U_{0}$. The collection $\left\{U_{s}: s \in T\right\}$ forms an open covering of $T$. Let $s_{1}, \ldots, s_{n}$ in $T$ be such that $T=\bigcup_{i=1}^{n} U_{s_{i}}$ and $\left\{f_{i}\right\}_{i=1}^{n}$ the partition of unity subordinate to $\left\{U_{s_{i}}\right\}_{i=1}^{n}$. Take $\beta_{c}=\sum_{i=1}^{n} f_{i} \cdot \beta_{s_{i}}$. A simple calculation will show that $\beta_{\varepsilon}$ satisfies (2.12) and (2.13). Now, for $s \neq t$, let $y_{\varepsilon} \in G$, be such that $\left\|\alpha(s)-y_{a}\right\|_{,} \leqslant r$ and $\left\|y_{d}-\beta(s)\right\|_{0}<$ $\varepsilon\left(y_{s} \in B(\alpha(s), r) \cap B\left(\beta_{\varepsilon}(s), \varepsilon\right) \cap G_{s}\right)$. Let $a_{s}=\left(y_{s}+\beta_{\varepsilon}(s)\right) / 2$ and $\Omega, \Omega_{s} \in G_{\infty}$ such that $\Omega(t)=x_{t}$ and $\Omega_{s}(s)=a_{s}$. Put $\gamma_{s}=(1-f) \Omega+f \Omega_{s}$ (where $f: T \rightarrow[1,0]$ such that $f$ is continuous, $f(t)=0$ and $f(s)=1)$. Let $U$, be the neighbourhood of $s$ such that $\left\|\alpha(p)-\gamma_{s}(p)\right\|_{p}<r+\varepsilon / 2$ for each $p$ in $U_{\text {. . Again }}\left\{U_{s}: s \in T\right\}$ forms an open covering of $T$. Let $s_{1}, \ldots, s_{n}$ in $T$ be such that $T=\bigcup_{i=1}^{n} U_{s_{i}}$ and $\left\{h_{i}\right\}_{i=1}^{n}$ the partition of unity subordinate to $\left\{U_{s_{i}}\right\}_{i=1}^{n}$. It can easily be checked that $\gamma_{e}=\sum_{i=1}^{n} h_{i} \cdot \gamma_{s_{i}}$ satisfies (2.12) and (2.14). Now, apply (2.12)-(2.14) inductively to construct a sequence $\left\{\gamma_{n}\right\}$ in $G_{\infty}$ with the following:

$$
\alpha_{n}(t)=x_{t},\left\|\alpha-\gamma_{n}\right\|_{\infty} \leqslant r+2^{-n} \text { and }\left\|\gamma_{n}-\gamma_{n+1}\right\|_{\infty} \leqslant 2^{-n} .
$$


The third inequality on the right in (2.15) implies that $\left\{\gamma_{n}\right\}$ is Cauchy, and then it has a limit $\gamma$ in $G_{\infty}$. Clearly $\gamma$ is the desired element.

Proof of THE THEOREM: Let $\left\{\alpha_{n}\right\}$ be a sequence in $E_{\infty}$ converging to $\alpha$, and $\beta$ an element in $P_{G_{\infty}}(\alpha)$. Define $r=d\left(\alpha, G_{\infty}\right)$ and $r_{n}=d\left(\alpha_{n}, G_{\infty}\right)$. For each $t \in T$ and $n \in N$, define $\mathcal{A}_{t}^{n}=B\left(\beta(t), d_{n}\right) \cap B\left(\alpha_{n}(t), r_{n}\right) \cap G$ (where $d_{n}=$ $\left.\left\|\alpha_{n}-\alpha\right\|_{\infty}+\left|r_{n}-\tau\right|+1 / n\right)$.

$$
\begin{aligned}
\left\|\beta(t)-\alpha_{n}(t)\right\|_{t} & \leqslant\|\beta(t)-\alpha(t)\|_{t}+\left\|\alpha(t)-\alpha_{n}(t)\right\|_{t} \\
& \leqslant r+\left\|\alpha_{n}-\alpha\right\|_{\infty} \\
& <r_{n}+\left|r_{n}-r\right|+\left\|\alpha_{n}-\alpha\right\|_{\infty}+\frac{1}{n} .
\end{aligned}
$$

By (2.16) $\mathcal{A}_{t}^{n}$ is a nonempty convex set for all $t \in T$ and for all $n \in N$. Now, let $t$ be an arbitrary but fixed element in $T$ and $n \in N$. Pick $x_{n} \in \mathcal{A}_{t}^{n}$. Let $\beta_{t}^{n} \in P_{G_{\infty}}\left(\alpha_{n}\right)$ be the element that exists from Lemma 2.6; that is, $\beta_{t}^{n}(t)=x_{n}$ and $U_{t}$ is the neighbourhood of $t$ such that $\left\|\beta_{t}^{n}(s)-\beta(s)\right\|_{s}<d_{n}$ for each $s \in U_{t}$ (such $U_{t}$ exists by the (u.s.c.) of the norm function). Thus for each $s$ in $U_{t}$ we have $\beta_{t}^{n}(s)$ in $\mathcal{A}_{s}^{n}$. The collection $\left\{U_{t}: t \in T\right\}$ is an open covering of $T$. Let $t_{1}, \ldots, t_{n}$ in $T$ be such that $T=\bigcup_{i=1}^{n} U_{t_{i}}$ and $\left\{f_{i}\right\}_{i=1}^{n}$ the partition of unity subordinate to $\left\{U_{t_{i}}\right\}_{i=1}^{n}$. Define $\beta_{n}=\sum_{i=1}^{n} f_{i} \cdot \beta_{t_{i}}^{n}$. For each $t$ in $T \beta_{n}(t)$ is a convex combination of elements of $\mathcal{A}_{t}^{n}$, and hence $\beta_{n}(t) \in \mathcal{A}_{t}^{n}$. Thus for each $t$ in $T$ we have

$$
\left\{\begin{array}{l}
\left\|\beta_{n}(t)-\alpha_{n}(t)\right\| \leqslant r_{n} \\
\left\|\beta_{n}(t)-\beta(t)\right\|<d_{n} .
\end{array}\right.
$$

The first inequality in (2.17) implies that $\beta_{n} \in P\left(\alpha_{n}\right)$ and the second implies that $\beta_{n} \rightarrow \beta$ as $n \rightarrow \infty$.

\section{REFERENCES}

[1] E. Behrends, $M$-structure and the Banach Stone Theorem: Lecture Notes in Math. 736 (Springer-Verlag, New York, 1979).

[2] E. Michael, 'Continuous selection', Ann. of Math. 63 (1956), 361-382. 